\title{
Finnougristik und Übersetzungswissenschaft ${ }^{1}$
}

1. Die Finnougristik ist ein Wissenschaftszweig, wo das Übersetzen und die Übersetzung von Anfang an eine wichtige Position innehatten: denken wir nur an die Veröffentlichung von Sprachproben oder an Untersuchungen, die sich mit linguistischen, folkloristischen oder ethnographischen Themen beschäftigen. Da das Übersetzen aus einer finnisch-ugrischen Ausgangssprache (AS) in der Regel in eine nicht-fiu. Zielsprache (ZS) geschah, vor allem ins Deutsche, waren Schwierigkeiten zu überwinden, deren Analyse Aufgabe der Übersetzungswissenschaft ist und aufschlussreiche Ergebnisse verspricht.

Gerade im Bereich der Finnougristik ist Jahrzehnte, bald Jahrhunderte übersetzt worden, ohne dass die an diesem Prozess beteiligten Sender wie Empfänger (Originatoren wie Rezipienten) je mit der Theorie der Übersetzungswissenschaft auch nur in Berührung gekommen wären oder diese in nennenswertem Ausmass vermisst zu haben. Die Frage der Lehrbarkeit und der Erlernbarkeit des Übersetzens soll hier auch nicht angeschnitten werden; sie ist selbstredend weitgehend zu trennen von dem, was man unter Übersetzungsübungen im Fremdsprachenunterricht in Schule und Hochschule versteht. Doch bringt die Bekanntschaft mit der Übersetzungswissenschaft auch einem erfahrenen Übersetzer zumindest eine Reihe von Aha-Erlebnissen und er fühlt sich dabei manchmal viel-

\footnotetext{
'Erweiterte Fassung eines Vortrages ('"Fennougristiikka ja käännöstiede"), den die Verf.in am 20. 1. 1978 auf der Versammlung der Finnisch-ugrischen Gesellschaft in Helsinki hielt.
} 
leicht dem Schriftsteller vergleichbar, dem der Literaturwissenschaftler durch seine Interpretation erst nachweist, was er sich eigentlich alles gedacht hat, als er Charaktere, Situationen und Handlung in seinem Werk so formte und beschrieb, wie er es getan hat. Gewiss spielen Empirie, Praxis und translatorische Kompetenz des Einzelnen eine wesentliche Rolle. Erst wenn man eine gewisse Erfahrung hat im Umgang mit Texten im Rahmen des Translationsprozesses wird man sich gewinnbringend mit den Erkenntnissen der Übersetzungswissenschaft auseinandersetzen können. Nun sind ja die Übersetzungswissenschaftler normalerweise durch ihre translatorische Tätigkeit zur Beschäftigung mit der Theorie gekommen. Es ist eine interessante Erscheinung, dass die Übersetzungswissenschaft im deutschsprachigen Raum stark mit der Romanistik liiert war und ist (denken wir z.B. an die Namen Mario Wandruszka, Fritz Paepcke, Harald Weinrich, Katharina Reiss).

Meine Ausführungen im folgenden müssen zunächst die allgemeine Problematik des Übersetzens skizzieren, ehe spezielle Bezüge zur Finnougristik hergestellt werden können.

2. Als selbständiges Fach, an Universitäten und Hochschulen vertreten, ist die Übersetzungswissenschaft relativ neu; sie ist ein Teilgebiet der vergleichenden Sprachwissenschaft. Der im vergangenen Juni in Montreal abgehaltene Mammutkongress der Übersetzer zeigte, dass die USA, die Sowjetunion und die Tschechoslovakei führend sind auf diesem Gebiet. Abhandlungen, Untersuchungen über übersetzungswissenschaftliche Themen sind heute in verschiedenen Sprachen in so grosser Zahl vorhanden, dass man Gefahr läuft, den Wald vor Bäumen nicht mehr zu sehen. Besonders aufschlussreich sind aber auch die alten Zeugnisse von Leuten, die viel übersetzt haben, im deutschsprachigen Raum etwa Martin Luther, Schleiermacher (von dem übrigens der Terminus Übersetzungswissenschaft stammen soll), Humboldt, Schlegel, Schopenhauer, Nietzsche usw. Allein mit geistreichen Zitaten für und wider das Übersetzen und seine Besonderheiten liessen sich ganze Bücher füllen. Fritz Güttinger bringt am Ende seines Buches "Zielsprache", statt einer Zusammenfassung ein solches Zitatengespräch, das er dann wohlmeinend mit Goethe ausklingen lässt: 'Was man 
auch von der Unzulänglichkeit des Übersetzens sagen mag, so ist und bleibt es doch eines der wichtigsten und würdigsten Geschäfte in dem allgemeinen Weltverkehr."

Man hat sich immer wieder mit der Frage der Übersetzbarkeit von Texten beschäftigt. Man hat die Möglichkeit des Übersetzens in Frage gestellt, hat das Ergebnis dieser Tätigkeit verglichen mit der Kehrseite eines Gobelins, das Übersetzen mit dem Umsetzen eines Musikstücks in eine andere Tonart, mit einem Kleiderwechsel u.a.m. Oder man hat bereits in der Etymologie der Verben und Nomina im Bereich der Translation vielsinnige Aussagen gefunden: ital. traduttore - traditore, fi. kääntää, ungar. fordítani 'umkehren, verdrehen'.

Lange Zeit wurde dem Übersetzer die Schuld an den misslungenen Übersetzungen bzw. an dem Unbehagen gegenüber Translationen gegeben; neuerdings konzentriert man sich dabei auf das sprachliche Material selbst, auf die Besonderheiten der jeweiligen Ausgangs- und Zielsprache, auf die sprachenpaarbezogene translatorische Performanz und untersucht die Gesetzmässigkeiten des Verstehens und Reproduzierens der Sprache überhaupt.

In Anbetracht der Kürze der Zeit muss die Frage der Übersetzbarkeit kategorisch beantwortet werden:

als sprachphilosophisch-erkenntnistheoretisches Problem positiv

als linguistisches Problem - positiv

als belletristisch-ästhetisches Problem - negativ als Textgattungsproblem - entweder positiv oder negativ.

Jean Pauls Wort 'Ein Kunstwerk, das einer Übersetzung fähig ist, ist keiner wert" kann ein Antonym von Ernest Renan gegenüberstellt werden: "'Ein nicht übersetztes Werk ist nur halb publiziert." Ein belletristischer Text, ein Text der sog. schönen Literatur, ist ein subjektives Kunstwerk; seine Übersetzung ist weitgehend künstlerische Produktion. Bei der Erforschung der subjektiven und objektiven Faktoren des Translationsprozesses lässt die Übersetzungswissenschaft schönliterarische und dichterische (lyrische) Texte normalerweise völlig ausser acht. Denn der Sprachgebrauch des Schriftstellers und 
Dichters weicht häufig von der Norm ab und die Aufgabe der Sprache ist in diesen Texten eine andere als im Sachtext. In der Fachliteratur ist die Sprache ein Kommunikationsmittel, während sie in der Belletristik gleichzeitig Gegenstand (Objekt) der Kommunikation ist, wobei die assoziativen und emotiven Komponenten der Sprache im Vordergrund stehen, indem auch die Metaphern, Wortspiele und onomatopoetischen Effekte bedeutungstragend sind. Die Konnotationen der einzelnen Wörter spielen in literarischen Texten eine andere Rolle als in Fachtexten; für erstere mag Harold Weinrichs Satz gelten: Übersetzte Wörter lügen immer, übersetzte Texte nur, wenn sie schlecht übersetzt sind (in "Linguistik der Lüge", Heidelberg 1966).

Die Aufgabe des Translators im Translationsprozess besteht darin, einen ausgangssprachlichen Text in einen funktionaläquivalenten Text einer bestimmten Zielsprache zu bringen. Seine translatorische Kompetenz besteht neben der Beherrschung der Ausgangs- und Zielsprache sowie deren aussersprachlicher Wirklichkeiten in der Kenntnis der Äquivalenzbeziehungen zwischen AS und ZS und der Fähigkeit, diese für die funktionale Äquivalenz konkreter Texte auszunutzen. Der politische, religiöse, soziale und dialektgeographische Hintergrund des Übersetzers beeinflusst seine Tätigkeit. Er muss seine Zielgruppe kennen, deren intellektuelles Niveau und deren Aufnahmefähigkeit, wobei es leicht zu Über-oder auch zu Unterschätzungen kommt.

3. Wenn man den Translationsprozess schematisch darstellen will, spricht man von den folgenden drei Phasen:

1. Dekodierung des AS-Textes (Analyse des Textes usw.)

2. Kodierungswechsel, Umkodierung, d.h. Umschlüsselung der dekodierten AS-Zeichenfolge in eine ZS-Zeichenfolge als spezifische Leistung des Translators

3. Rekodierung des ZS-Textes als Endprodukt (Translat), d.h. hier wird der Translator zum uneigentlichen Sender (Sender 2) und kommuniziert mit dem Zweitempfänger (der Erstempfänger in Phase 1 war der Translator).

Phase 2 ist also besonders relevant; diese Umkodierung zeigt, dass es sich beim Übersetzen nicht um einen einfachen Austausch verschiedensprachlicher Zeichen handelt. Formale Ele- 
mente wie grammatische oder syntaktische Strukturen sind in der Translation variant. Auf der Inhaltsebene muss trotz Kodierungswechsel die Invarianz gewahrt bleiben.

Die Frage der maschinellen Übersetzung soll hier nur gestreift werden. Der Computer als Datenbank, als Speicher von naturwissenschaftlich-technischen Terminologien ist eine Tatsache. Ansonsten ist man heute von der anfänglichen Euphorie abgekommen, die einmal den Computer an die Stelle des Humantranslators treten sah. Schon im 17. Jh. war es Johann Joachim Becker und im darauffolgenden Jahrhundert u.a. Gottfried Wilhelm Leibniz, die ein System der Metasprache entwickelten, um eine mechanische Übersetzung anzufertigen. Die Schwächen und Schwierigkeiten des Systems waren damals wie heute gleich: der Computer übersetzt ungenauer und langsamer als der Mensch, die Vor- und Nachbereitung bei der maschinellen Übersetzung ist so zeit- wie kostenaufwendig, die idiomatischen Wendungen und die Komposita bilden eine besonders grosse Fehlerquelle. Alle einschlägigen Werke liefern Beispiele für entsprechende Fehlleistungen des Computers. Ich möchte die Problematik, um die es bei der maschinellen Übersetzung geht, durchaus nicht simplifizieren, doch finde auch ich das folgende Beispiel zu aufschlussreich, um es weglassen $\mathrm{zu}$ können. Es geht um die maschinelle Übersetzung des in vielen Sprachen belegten Sprichwortes "Aus den Augen, aus dem Sinn" (fi. poissa silmistä, poissa sydämestä, engl. out of sight, out of mind). Der Computer übersetzte dieses englische Sprichwort ins Russische. Als er dann die Aufgabe erhielt, das russische Translat ins Englische rückzuübersetzen, lautete der Output: "invisible idiot"!

Das Übersetzen ist keine mechanische Tätigkeit, es ist ein schöpferischer Prozess. Wir wissen heute noch viel zu wenig darüber, wie der Mensch übersetzt. Wenn wir erfahren werden, wie das eigentliche Übersetzen vor sich geht, wenn das Zeichensystem der Ausgangssprache durch das der Zielsprache ersetzt wird, könnten wir das Modell dieses Prozesses beschreiben und für die Maschine auswerten. Die Eigenschaften der natürlichen Sprachen wie Redundanz, Homonymie, Polysemie, Synonymie, Strukturvielfalt, die unterschiedliche Seg- 
mentierung der Welt durch die einzelnen Sprachen lassen verstehen, dass der Humantranslator durch keine Maschine wird ersetzt werden können.

Grob verallgemeinernd kann man zwei verschiedene Methoden des Übersetzens unterscheiden. Das hat bereits Schleiermacher Anfang des 19. Jh. folgendermassen formuliert: 'Entweder der Übersetzer lässt den Schriftsteller möglichst in Ruhe und bewegt den Leser ihm entgegen, oder er lässt den Leser möglichst in Ruhe und bewegt den Schriftsteller ihm entgegen. Beide [Wege] sind so gänzlich voneinander verschieden, dass durchaus einer von beiden so streng als möglich muss verfolgt werden, aus jeder Vermischung aber ein höchst unzuverlässiges Resultat nothwendig hervorgeht, und $\mathrm{zu}$ besorgen ist, dass Leser und Schriftsteller sich gänzlich verfehlen." Seine Methode 1 ist die des Verfremdens, der Erweiterung und Neuerwerbung von Sprachraum; ihre Grenze liegt für die literarische Übersetzung in der Verstehbarkeit. Methode 2 ist die des Adaptierens, des Eindeutschens, ihre Grenze liegt bei der stilistischen Eigenart des Textes.

4. Was zwischen dem Verstehen des Originals und der ersten gedachten Übersetzung geschieht, pflegt man als Übersetzungsprozedur oder -verfahrensweise zu bezeichnen. Hinsichtlich ihrer Anzahl und Benennungen werden verschiedene Auffassungen vertreten. Die wichtigste Einteilung ist wohl die in wörtliche und nicht-wörtliche Übersetzung. Untergruppen der ersteren Art sind 1. Lehnübersetzungen, 2. Wort-für-WortÜbersetzungen und 3. wörtliche Übersetzungen (Substitution). Minä olen lukenut kirjan 'ich habe das Buch gelesen' ist eine wörtliche, aber keine Wort-für-Wort-Übersetzung.

Nicht-wörtliche Übersetzung besteht aus den Untergruppen 1. Modulation (Veränderungen des Wortinhalts gemäss der Zielsprache, vgl. fi. matala, dt. untief) und 2. Transposition (Translokation) (Umwandlung grammatischer und syntaktischer Elemente, vgl. kehittyvä yhteiskunta 'die Entwicklung der Gesellschaft').

Es hängt von der typologischen Verwandtschaft der am Translationsprozess beteiligten Sprachen ab, welche Übersetzungsmethode vom Standpunkt der Kommunikation jeweils die bestmögliche ist. 
Bei der Beurteilung übersetzter Texte bedient man sich häufig der Unterscheidung: wörtliche Übersetzung - freie Übersetzung. Diese Grenze ist aber durchaus nicht eindeutig und im Einzelnen sehr schwer zu ziehen. Im Prinzip gibt es davon soviel Grade oder Abstufungen wie die entsprechende Textstelle Ebenen hierarchischer Strukturen aufweist ${ }^{1}$.

5. Bedauerlicherweise herrscht auch in der Übersetzungswissenschaft keine Klarheit und Eindeutigkeit in der Verwendung der Terminologie, der Fachausdrücke. Fast jede linguistische Schule entwickelt ihre eigene Übersetzungstheorie. Die Uneinheitlichkeit der Termini zeigt sich besonders auch in der Charakterisierung der Textsorte, -gattung, -art, jener primären Variablen, von der der Übersetzer auszugehen hat. Den einzelnen Textsorten sind funktionale Stilmittel eigen, die einmal von bestimmten Normen abhängen und zum andern charakteristisch sind für die Kommunikationssituation. K. Reiss unterscheidet den inhaltsbezogenen Texttypus (neuerdings nennt sie ihn informativen Typ) (eigtl. Fachliteratur, wie Abhandlungen, Sachbücher, Reisebeschreibungen, Rezensionen usw.), den formbetonten Texttypus (expressiver Typ) (literarische Texte), den appellbetonten Texttypus (operativer Typ) (Werbe-, Propagandatexte u.a.) und den audio-medialen Texttyp (Hörspiele etc.).

1 Vgl. die Ausführungen zweier Übersetzungswissenschaftler: "The terms 'literal' and 'free' thus do not really form a clear binary contrast. A word-byword rendering is literal as compared with a loose translation of a whole sentence, but free as compared with a morpheme-by-morpheme rendering. It may be preposed that, for any given passage, there are as many degrees of literalness and freedom of translation as there are levels of hierarchical structures in the passage". (C. F. Hockett, "Translations via immediate constituents" in: IJAL 20, S. 313.) "A common distinction is often made between literal or word-for-word translation and idiomatic or free translation. But there are more than just two degrees on the scale of literalness and idiomaticity. If we go below the level of the word, there can also be morpheme-by-morpheme translation, while if one tries to translate proverb by proverb, there is often no corresponding internal structure at all'. (Y. R. Chao, "'Translation without Machine" in: H. G. Lunt [Hrsg.], Proceedings of the Ninth Intern. Congress of Linguists, Cambridge/Mass. 1962. Den Haag 1964, S. $507 \mathrm{f}$.) 
Es gibt aber auch multifunktionale Textsorten, die - abhängig vom Kontext - verschiedenen Texttypen angehören können. Wichtig ist ferner, dass die ursprüngliche kommunikative Funktion eines Textes in einigen Fällen unbekannt ist, was sich z.B. durch die Entfernung in Raum und Zeit zwischen Textproduktion und Translation ergibt. Diese Erscheinung ist z.B. beim Übersetzen von Sprachproben zu linguistischen Zwecken von Bedeutung. Solche Sprachproben können natürlich nur dann adäquat übersetzt werden, wenn die Situation des Aufzeichnens, die Funktion des Textes, die Einstellung von Informant und Sammler usw. bekannt sind. - Ferner ist jeder konkrete Text normalerweise eine asystematische Kombination diverser Einzeltypen, worin die eigentliche Kernproblematik denn auch verborgen liegt.

Die Länge des Textes ist kein unwesentlicher Faktor; Textumfang und Schwierigkeitsgrad des Übersetzens sind umgekehrt proportional. Kleinste Texteinheiten können somit leicht unübersetzbar anmuten, man denke hier etwa an Wörterbücher kleinerer fiu. Sprachen mit Beispielmaterial, das über ein Einzelwort hinausgeht.

Die Textanalyse kann vor dem eigentlichen Übersetzungsprozess erfolgen, wobei Verstehensphase und Übersetzungsphase aufeinander folgen. Beide können auch miteinander verschmelzen, wie es z.B. bei der Arbeit der Simultandolmetscher der Fall ist. Claus Catellieri hat in seiner Dissertation "Zur Analyse des Ausgangstextes beim Übersetzen"' (Beiträge zu einer angewandten Semiotik; Leipzig 1970) auf die eminente Bedeutung der Arbeit am Ausgangstext für die Theorie und Praxis der Übersetzungswissenschaft hingewiesen. Es geht ihm um die Charakterisierung von Originator und Rezipient im Relationsgefüge der jeweiligen Kommunikationssituation und die dabei feststellbaren Verflechtungen. Er hat einen ganzen Komplex von Fragen ausgearbeitet, die sich der Übersetzer zu stellen und die er zu beantworten hat, ehe er mit der eigentlichen Arbeit beginnt.

Auf der anderen Seite wird immer wieder darauf hingewiesen, wie die Beschäftigung mit der Muttersprache, d.h. die Ausbildung in - im Normalfall - der Zielsprache vernachlässigt wird. 
Eine mangelnde Beherrschung der Muttersprache bildet eine häufigere Fehlerquelle als man allgemein vermutet. Darauf weist u.a. Lóránt Tarnóczi hin, der sich in Ungarn höchst verdienstvoll mit der Übersetzungswissenschaft beschäftigt hat und dem wir wichtige Publikationen zu diesem Thema verdanken. ${ }^{1}$

7. Besonders wesentlich erscheint mir die Beschäftigung mit der linguistischen Stilistik, wie sie von Elise Riesel ausgearbeitet worden ist. Sie geht von 4 Stilebenen oder -schichten aus: Gehobener Stil (mit dichterischem Stil als Teilebene), Normalstil bzw. Normalsprache ${ }^{2}$, Saloppe Umgangssprache und Vulgärsprache. Im Bereich der Normalsprache unterscheidet sie die funktionalen Stile der Wissenschaft, der Presse und Publizistik, des öffentlichen Lebens, der Alltagsrede und der schönen Literatur. Und um diesen funktionalen Stil der Wissenschaft geht es, wenn man fachwissenschaftliche Texte übersetzt, wie es im Rahmen der Finnougristik geschieht. Diesen Stil der Wissenschaft müsste man nun untergliedern je nach den Disziplinen, ihn abtasten auf seine lexikalischen und syntaktischen Besonderheiten und zwar - im Hinblick auf das Übersetzen - sprachenpaarbezogen.

Eine übersetzungswissenschaftlich orientierte konfrontative Stiltypbeschreibung führt zu beachtlichen Ergebnissen hinsichtlich der stilistischen Äquivalenzbeziehungen, wie es Heide Schmidt in ihrem Aufsatz "Probleme der konfrontativen Be-

1 U.a.: Fordítókalauz. A szakirodalmi fordítás elmélete és gyakorlata. (Führer für den Übersetzer. Theorie und Praxis der fachsprachlichen Übersetzung.) Budapest 1966; ders., Fordítástechnika (Müszakigazdasági fordítások idegen nyelvböl magyarra) (Übersetzungstechnik. Die Übersetzung von technisch-wirtschaftlichen Texten aus Fremdsprachen ins Ungarische.) Budapest 1972. (Inhalt und Zusammenfassungen auch in Englisch, Russisch und Deutsch.)

2 Im neuesten und besten normativen Wörterbuch der deutschen Sprache, dem auf 6 Bände veranschlagten, vom Duden-Verlag in Mannheim publizierten Lexikon namens "'Das grosse Wörterbuch der deutschen Sprache' (erschienen sind bislang 4 Bände) wird als Teilebene der Normalsprache noch die Bildungssprache unterschieden, der z.B. Wörter angehören wie dt. Affront, analog, eruieren. 
schreibung stilistischer Selektionsbeschränkungen" anhand von Zeitungskurzmeldungen bewiesen hat ${ }^{1}$. Als Komparabilitätskriterien in einer $L_{1}$ und einer $L_{2}$ dient die stilistische sowie semantisch-denotative Information. Ein solcher funktionaler Stiltyp setzt sich aus zwei unterschiedlichen Formen von Selektionsbeschränkungen zusammen: 1. aus absoluten Restriktionen, durch die bestimmte sprachliche Elemente oder Strukturen in einem bestimmten Stiltyp ausgeschlossen sind und 2. aus Häufigkeitsrestriktionen, die für die Frequenz eines Stilelements in einem Stiltyp zuständig sind. Diese zweite Gruppe ist von immenser Bedeutung für die Übersetzungswissenschaft, denn die Nichteinhaltung dieser unterschiedlichen Beschränkungen in der $\mathrm{L}_{1}$ und der $\mathrm{L}_{2}$ ist eine der Hauptquellen der Interferenzen in Übersetzungen.

Fachsprachliche Texte haben vor allem eine horizontverändernde, nicht situationsverändernde Aufgabe (Brinkmann).

Die logische Normativität des aussersprachlichen Sachverhalts hat translatorische Normativität zur Folge. Und hier liegt ein wesentlicher Unterschied zu literarischen Texten: das Prinzip der Mehrfachübersetzbarkeit eines und desselben Textsegments gilt für Fachtexte nicht (jedenfalls nicht auf lexikalischer Ebene; natürlich wird auch der Autor eines Fachtextes stilistische Eigenheiten haben, die in der ZS wiederzugeben sind). Die Rolle des Synonyms im literarischen Text ist eine ganz andere als die der terminologischen Doublette im Fachtext.

Paepcke spricht vom "Gesetz der terminologischen Distanz", das Sender und Empfänger als subjektive Faktoren des fachsprachlichen Textherstellungs- und Textverstehensprozesses gewissermassen ausschliesst. Auch syntaktisch und syntagmatisch sind fachsprachliche Texte weitgehend stereotyp; man spricht von "habituell verfestigten Vertextungsstrategien" (Plett), die relativ leicht erkennbar und damit beschreibbar unc auf diesem Wege auch lehrbar bzw. erlernbar sind.

\footnotetext{
1 Veröffentlicht in: Vermittelte Kommunikation, Sprachmittlung,Translation. Übersetzungswissenschaftliche Beiträge 1. Hrsg. Otto Kade. Leipzig 1977.
} 
Selbstverständlich verlangt das Übersetzen von Fachtexten auch Fachwissen und setzt darüber hinaus z.T. eine erhebliche und zeitraubende Beschäftigung mit der in der ZS vorhandenen diesbezüglichen Fachliteratur voraus.

8. Ein wesentlicher Bestandteil der Übersetzungswissenschaft ist die Übersetzungskritik, der Übersetzungsvergleich. Man vergleicht das Original mit der Übersetzung, mit der Übersetzung in verschiedene Sprachen oder man vergleicht Übersetzungen, die verschiedene Übersetzer vom selben Original angefertigt haben. Man untersucht und weist nach, wie übersetzungstechnische Schwierigkeiten im Bereich der Semantik, Syntax, Stilistik u. Pragmatik gelöst worden sind. Dadurch erhält man 1. eine Typologie der Übersetzungsschwierigkeiten für verschiedene Texte, Textsorten und Sprachen (ein Desideratum übrigens immer noch für Fi.-Dt., Dt.-Fi. ${ }^{1}$ ), 2. diverse Methoden zur Überwindung von Übersetzungsschwierigkeiten und zum Auffinden von Äquivalenzen, 3. Bewertungskriterien für verschiedene Texttypen, 4. neues Äquivalenzmaterial zur Erweiterung der Wörterbücher.

Übersetzungskritik sollte aber nicht nur auf dieser Ebene geschehen, sondern bereits im Verlag durch Verlagslektoren. Da dies Thema in Finnland ein wunder Punkt ist, möchte ich an dieser Stelle darüber schweigen.

Erfreulicherweise hat man in letzter Zeit in der Presse im Zusammenhang mit Buchbesprechungen auch so etwas wie Übersetzungskritik geübt. Dass es gerade auch für Fachliteratur eine entsprechende Kontrollinstanz vor der Veröffentlichung von Übersetzungen geben müsste, liegt auf der Hand.

9. Die Finnougristik ist ein dankbares Gebiet für die Übersetzungswissenschaft; die spezifischen Forschungsthemen

1 Durch die von János Juhász in Ungarn durchgeführten oder initiierten Untersuchungen im Bereich der Interferenz weiss man mehr über die Beziehungen Dt.-Ungar., Ungar.- Dt. (vgl. bes. János Juhász, Probleme der Interferenz, München 1970). Allerdings verdient auch die viel zu wenig bekannte Arbeit von Hans Martin Erwähnung und Beachtung: "Untersuchungen zur sprachlichen Interferenz auf der Grundlage finnlanddeutschen Materials"' (Annales Universitatis Turkuensis. B. 129. Turku 1973). 
können hier höchstens beim Namen genannt werden. Es handelt sich dabei einmal um die kontrastive Lexikologie und die kontrastive Semantik. Man müsste jene an den sprachlichen Kontext gebundenen Subsysteme erforschen, die die Definition eines jeden Wortfeldes bewirken. Durch Abgrenzung und Identifizierung der verschiedenen Varianten des Sprachgebrauchs wird die Erklärung der konkurrierenden Bedeutungen ein und desselben Lexems erleichtert. Durch innersprachliche Hierarchie und aussersprachliche Gebrauchsvarianten erklären sich die semantischen Divergenzen.

Wie aus den obigen Ausführungen hervorging, spielt auch die Umstrukturierung der Syntax beim Übersetzen eine bedeutende Rolle. Die Verschiebungen im Verhältnis zwischen Semantik und Syntax in den einzelnen am Translationsprozess beteiligten Sprachen kann nach Cartellieri als Differenzierung zwischen Produktion und Reduktion interpretiert werden. In einem Satz wie fi. Hän puhuu aina huonosta voinnistaan 'er redet a ber a ch immer davon, wi e schlecht es ihm geht' verlangt die syntaktische Reduktion bei dieser Übersetzungsrichtung eine semantische Produktion.

Die sog. pragmatische Übersetzungswissenschaft strebt danach, Hilfsmittel zu schaffen und/oder zu verbessern, die der Übersetzer in seiner praktischen Arbeit benötigt. Ein- und mehrsprachige Wörterbücher, auch Fachterminologien, sind bereits zahlreich vorhanden; allerdings übersieht der Übersetzer mitunter, dass dies ja keine Übersetzungswörterbücher sind, das könnte nur ein Satzlexikon sein, als Hilfsmittel aber unökonomisch und seines Umfangs wegen schwierig zu handhaben. Im Bereich der Finnougristik fehlen vorläufig (ausser für das Ungarische) eigentliche Synonymwörterbücher, es fehlen umfangreiche Frequenzwörterbücher, speziell fachbezogene, wie man sie heute in den sog. Weltsprachen immer mehr zusammenstellt. Der spürbarste Mangel dürfte das Fehlen idiomatischer Wörterbücher sein, besonders der vergleichenden Idiomatik.

Bei den wissenschaftlichen Publikationen, die z.B. aus dem Finnischen oder Ungarischen ins Deutsche übersetzt sind (ich beschränke mich hier auf die Finnougristik und dabei speziell 
auf Sachvolkskunde, Folkloristik u. Linguistik) fallen besonders drei Besonderheiten auf, alle im Bereich der Lexik: 1. Frage der Tradition, 2. Rolle der Neubildung, 3. Ausfüllung der lexikalischen Lücken i.a.

Charakteristisch für jede Fachsprache ist ihr spezieller Wortschatz. Zwischen der nichtfachbezogenen Normallexik und der Fachlexik liegt eine Übergangszone mit restriktiver Verständlichkeit. Seit Bestehen der Wissenschaft der Finnougristik sind die meisten Publikationen auf diesem Gebiete ins Deutsche übersetzt worden. An der Herausbildung der Terminologie waren einige wenige sehr produktive und namentlich bekannte Übersetzer beteiligt. Wer nun heute finnougristische Arbeiten übersetzt, muss diese Tradition berücksichtigen. Will er erneuern, was manchmal durchaus angebracht erscheint, gerät er in Konflikt mit den jeweils zu zitierenden Quellen, worunter die Folgerichtigkeit leidet, die in der wissenschaftlichen Terminologie eine conditio sine qua non ist.

Es gibt immer wieder Fälle, wo sich diese Tradition als eine Last erweist: wenn ostj. iki bislang immer durch 'Greis' (pro in casu - '(alter) Mann, Alter'), ostj. jor durch 'Wirt' (pro - in casu - 'Mann; (Haus)herr, (Familien)vater' etc.) übersetzt worden ist, kann man nicht ohne weiteres auf diesen Brauch verzichten, auch wenn die Gegenwartssprache und besonders die jeweilige Kommunikationssituation im Kontext dies verlangt. Aufschlussreich sind die diesbezüglichen Anmerkungen von W. Steinitz in seiner Arbeit "Ostjakische Volksdichtung und Erzählungen aus zwei Dialekten" (vor allem in Band II), wie die traditionelle Übersetzung den Text oft undeutlich macht, obwohl auch er keinen anderen Weg sieht, als diese zu verwenden und in der Anmerkung z.B. über das ostj. Wort $w \bar{\jmath} \check{s}$ zu schreiben: "die traditionelle Übersetzung 'Stadt' ist nicht sehr glücklich, $w$.ǰs bedeutet 'grössere, als Mittelpunkt dienende Siedlung'."

Es wäre verlockend, der Bedeutung und der Rolle des Übersetzens und der Übersetzer in der Geschichte der Finnougristik einmal genauer nachzugehen. Dazu ist in dieser kurzen Darstellung jedoch keine Gelegenheit. Ein Name darf allerdings nicht ungenannt bleiben: Gustav Schmidt, der in Finnland als 
deutscher Übersetzer der Finnougristik eine Tradition schuf. Seine translatorische Kompetenz war von erstaunlicher Vielseitigkeit, denn er übertrug neben Fachliteratur auch Belletristik (so hat er z.B. "Die sieben Brüder" und " Die Heideschuster" von Aleksis Kivi übersetzt). Seine sichere Sachkenntnis, gepaart mit einer guten Beherrschung der AS und ZS, befähigte ihn, als Sprachmittler schöpferisch zu werden und eine finnougristische Terminologie im Deutschen zu schaffen, auf die sich die späteren Übersetzer verlassen und stützen, die sie als Ausgangspunkt benutzen konnten.

Der Übersetzer muss sich häufig als eine Art Sprachpfleger verstehen, der ein subtiles Gespür für Interferenzen hat. Wahrscheinlich hat man die Interferenzen bislang zu negativ, d.h. einseitig bewertet; so mehren sich in der letzten Zeit denn auch die Stimmen, die auf die erweiternde und bereichernde Bedeutung und Funktion der Interferenz hinweisen. E. Coseriu geht - m.E. mit Recht - sogar so weit, die sprachliche Interferenz als eine Form der sprachlichen Kreativität anzusehen: sie sei dem innersprachlichen Schaffen analog. Interferenz könne durchaus korrekte Produkte bewirken, korrekt im Sinne des Sprachsystems und der Sprachnorm. Besonders interessant ist hier auch die Erscheinung der Nicht-Realisierung ("negative Realisierung"'): gerade der gebildete Sprachträger vermeidet es häufig intentionell, die den interferierenden Sprachen gem e in s a m e n Ausdrucksmöglichkeiten zu verwenden. ${ }^{1}$

Andererseits ist aber auch festzustellen, dass die Toleranzschwelle für nichtkorrekte Realisierungen der Zielsprache erstaunlich niedrig liegt, abhängig wohl davon, dass der sprachliche Normalverbraucher seiner Muttersprache nicht reflektierend begegnet. Man kann das immer wieder an Leuten beobachten, die neu nach Finnland kommen: kaum sind sie im Lande normalerweise weder des Schwedischen noch des Finnischen mächtig - schon sprechen sie vom "Wachtmeister" (fi. vahtimestari, schwed. vaktmästare) im Sinne von - je nachdem -

I Vgl. die Aufsätze von Eugenio Coseriu und Mario Wandruszka in der Festschrift für Werner Betz "Sprachliche Interferenz", Tübingen 1977. 
Hausmeister, Pedell, Kirchendiener oder etwa vom "'Studentexamen" (das schwedische Wort wird übernommen) statt von Reifeprüfung oder Abitur. - Diese Toleranzschwelle ist aber auch gerade bei Wissenschaftlern selbst, d.h. Vertretern des betreffenden Faches, oft sehr niedrig, indem sie weitgehend geneigt sind, in der Terminologie der Ausgangssprache entgegenzukommen, ganz in dem Sinne etwa, wie Ortega y Gasset es seinerzeit postulierte. Als Begründung wird häufig angeführt, dass es sich bei einem solchen Terminus ja um die Bezeichnung einer territorialen Besonderheit handle, welches Argument gar nicht immer stichhaltig ist. Ein Beispiel aus der Sachvolkskunde: fi. kaappikello '(Boden)standuhr', fi. kellokaappi 'Uhrenkasten, Gehäuse' wurde von deutschen Ethnologen auch in der Übersetzung 'Schrankuhr', 'Uhrenschrank' gutgeheissen, ja, diese wörtliche Übersetzung wurde sogar der idiomatischen deutschen vorgezogen.

Bei der Ausfüllung lexikalischer Lücken in der Zielsprache unterscheidet man mehr als ein Dutzend Methoden, angefangen vom Zitatwort über Lehnschöpfungen bis hin zu Teil- und Totalumschreibungen mit oder ohne Fussnote. Hier sei noch auf zwei Besonderheiten eingegangen. Einmal die Frage des Artikels im Deutschen. Welchen Artikel erhält ein finnischer Ausdruck oder Name, wenn er als solcher z.B. in deutschem Text verwendet wird? Strittig ist hierbei immer wieder das Kalevala. Über dieses Thema schrieb übrigens schon Gustav Schmidt (FUF II 1902). Seiner Feststellung nach hat man in alten Texten des 19. Jh. hierfür ausschliesslich den die-Artikel verwendet, einmal steht der. Er selbst plädiert für den das-Artikel: er appelliert an das Sprachgefühl und erinnert, dass der Artikel im Deutschen in solchen Fällen normalerweise nach der Bedeutung des Fremdwortes gegeben werde: das Kalevala als das Land od. Heim des Kaleva.

Diese Frage verdiente eine genauere Untersuchung. Denn die Wahl des Artikels wird auch von anderen Faktoren beeinflusst, z.B. der Analogie, dem Systemzwang. Doch gerade die Analogie ist hier nicht eindeutig: wie erklärt es sich z.B., dass ein Deutscher (normalerweise des Finnischen nicht kundig) durchweg der Helsingin Sanomat, die Mannerheimintie usw. 
sagt? Denn den Mustern mit der (Apparat, Automat, Soldat) stehen ebenso solche mit das (Dezernat, Konsulat, Zitat) sowie auch mit die (Heimat, Tat) gegenüber, wobei im Deutschen die letzte Silbe sogar noch einen langen Vokal enthält, was hierbei nicht ins Gewicht fallen dürfte. Ein Deutscher ist in diesem Fall ja meist nicht imstande, die Bedeutung des Namens z.B. "'Helsingin Sanomat" zu übersetzen; doch richtet er sich auch nicht nach der in deutschen Grammatiken stehenden Regel, eine Zeitung verlange in diesem Fall eben den die-Artikel.

Die zweite Besonderheit ist die Verwendung deutscher Dialektwörter, besonders solcher, die oft aus dem Finnischen ins Preussische gelangt sind, als Entsprechungen für finnische Ausdrücke. Eine Fundgrube entsprechender Dialektwörter ist das 1833 erschienene zweibändige "Preussische Wörterbuch" von H. Frischbier. Fi. talkoot pflegt als 'Gemeinwerk, Nachbarschaftshilfe' übersetzt zu werden, wenigstens im Bereich der Volkskunde. Im normalsprachlichen Kontext bieten sich ad hoc-Übersetzungen an. Frischbier hat "der Talk, die Talke; talken", deren Verwendung in einem deutschen Text ohne ausführliche Erklärung nicht verständlich und daher nicht empfehlenswert ist. Fi. kiisseli 'Beeren(süss)speise, eine Art rote Grütze' ist im Preuss. "'Kissel', $f$. und $m$. In der Belletristik ist die Verwendung solchen Sprachgutes u.U. angebracht, wenn nämlich der aussersprachliche Kontext dazu berechtigt.

Lexikalische Lücken werden weiter durch das textsemantische Verfahren ausgefüllt, das den Text erweitert oder verändert, sowie durch das textsyntaktische, das die Reihenfolge im Text, Satz oder Abschnitt ändert. Eine Besonderheit der Textsemantik stellen die Phraseologismen dar, die idiomatischen Wendungen. Ihre Übersetzung geschieht nach dreierlei Verfahren:

1. die wörtliche Übersetzung, z.B. fi. Ilta on aamua viisaampi 'Der Abend ist klüger als der Morgen': in der ZS entsteht ein mögliches Idiom;

2. die relativ entsprechende Übersetzung, in der ZS als Idiom geläufig: z.B. fi. Ei kukaan ole seppä syntyessään 'Es ist noch kein Meister vom Himmel gefallen'; ung. most ugrik 
a majom a vizbe 'Jetzt kommt der Moment, wo der Frosch ins Wasser rennt', ung. világos mint a vakablak 'klar wie dicke Tinte / Klossbrühe';

3. die erklärende Übersetzung, die keinen Phraseologismus in der ZS bildet, z.B. fi. Syytä on nïn sysissä kuin sepissäkin 'Beide Seiten haben schuld'.

10. Im folgenden soll ein finnischer Originaltext (Terho Itkonen, Lapin paikannimistön huoltoa [Die Pflege des Ortsnamengutes von Lappland]. In: Kalevalaseuran Vuosikirja 52 1972, 284-304, Textauszug S. 284/5) und seine deutsche Übersetzung unter dem Gesichtspunkt der Übersetzungsäquivalenz durchleuchtet werden.

Jene Stellen im Text, auf die besonders eingegangen wird, sind gekennzeichnet: fetter Druck gibt syntaktische, Kapitälchen semantische und Kursivdruck gebrauchsnormativ-funktionalstilistische Übersetzungsschwierigkeiten an. Alternative Übersetzungsmöglichkeiten sind in Klammern angeführt, aus Raummangel allerdings nur in Einzelfällen.

Ausgangssprachliche Textanalyse. Der Text, ein Stück Sachprosa, gehört der Normalsprache an und ist als inhaltsbezogen bzw. -betont ( = informativ) zu klassifizieren. Obwohl er funktional-inhaltlich als fachsprachlicher Text über ein Problem der Onomastik an sich der Wissenschaft (Sprachwissenschaft) zuzuordnen ist, könnte er hinsichtlich seiner denotativen Markiertheit und lexikalischen Selektionsbeschränkungen ebensogut als Stil des öffentlichen Lebens bezeichnet werden. Der Text ist ohne nennenswerten Informationsverlust übersetzbar, zwischen ausgangs- und zielsprachlichem Text besteht keine "'kommunikative Differenz" (Plett), und die Zahl der potentiellen Äquivalenzbeziehungen ist begrenzt.

Syntaktisch ist die Textstelle klar und relativ unkompliziert. Semantisch enthält der Text sowohl normal- als auch fachsprachliche Lexik.

Stilistisch wirkt der Text gerade durch seine sprachliche Ausgewogenheit trotz seiner typisch analytischen Struktur wohltuend einfach und echt; auffallend die häufigen unpersönlichen Verbalkonstruktionen, abhängig von der Textsorte. 


\footnotetext{
1 Ympäristönsuojelua koskevassa keskustelussa on

2 syrjitty Lappi alkanut nousta yhä pinnemmalle:

3 vaikka asenteet muuttuvat hitaasti,

4 on vähitellen herätty tajuamaan,

5 mitä Lapin maisemat merkitsevät Suomelle

6 sekä itseisarvona että asujiensa perinnäisenä elinympäristönä.

7 Noihin maisemiin kuuluu

8 ja ihmisen suhdetta niihin ilmentää myös paikannimistö.

9 Nimistön huoltoa käsiteltäessä

10 ei Lapin paikannimien vaalinta ole usein tullut puheeksi,

11 mutta kaikessa hiljaisuudessa on

12 tätäkin ympäristönsuojelun alaa meillä harrastettu,

13 erityisesti 1960-luvulta lähtien yhä ohjelmallisemmin ja tehokkaammin.

14 Tuloksia alkaa vähitellen näkyä julkisuudessa.

15 Lapin paikannimien pulmiin

16 on meillä vanhastaan joutunut ottamaan kantaa

17 ennen kaikkea maanmittaushallitus KARTOITUSTYÖSSÄÄN.

18 Valtavasti laajentunut Lapin-harrastus eri ilmauksineen

19 - tieteellinen tutkimus, luonnonvarojen hyödyntäminen,

20 uudisasutus, ERÄRETKEILY, massaturismi -

21 on tuonut LANTALAISET Lapin perimpiinkin sopukoihin

22 ja antanut samalla nimistön tuntijoille haasteen:

23 minkä nimisillä paikoilla ollaan liikkumassa?

24 Toisaalta on alettu oivaltaa

25 LAPIN HEIMON oikeus

26 omaan kieleensä, sen suojeluun ja KEHITTÄMISEEN.

27 Nimistönhuolto kuuluu tähän työhön luontaisena osana:

28 nimet edustavat sekä käytännön kielipääomaa

29 että tunteenomaisesti tajuttuja PERINNEARvOJA,

30 joiden käsittelystä ei selvitä olankohautuksella.
}

Übersetzungsschwierigkeiten und ihre Überwindung. Syntax. $\mathrm{Da}$ der Text keine grossen syntaktischen Schwierigkeiten enthält, ist im Rahmen der strukturellen Unterschiede beider Sprachen eine relativ wörtliche Übersetzung möglich. Es kommt zu Transpositionen, wie sie bei der Übersetzungsrichtung Finnisch $\rightarrow$ Deutsch üblich sind. Abhängig von der vorliegenden Textsorte haben wir in Zeile 2, 4, 14 und 24 der AS eine Verbalkonstruktion, deren inchoativer Aspekt im Deutschen am besten nur durch ein Adverb (allmählich, langsam, nunmehr) wiedergegeben werden kann. Im syntaktischen Be- 
1 In der Diskussion über den Umweltschutz tritt

2 das einst vernachlässigte Lappland heute immer mehr in den Vordergrund.

3 Die Einstellungen ändern sich zwar nur langsam,

4 doch hat man allmählich begriffen,

5 was die Landschaften Lapplands für Finnland bedeuten,

6 einmal als Selbstwert, zum andern als traditionelles Lebensmilieu ihrer Bewohner.

7 Auch die Ortsnamen gehören zu diesen Landschaften

8 und bringen die Beziehungen der Menschen zu ihnen zum Ausdruck.

9 Im Zusammenhang mit der Namenpflege

10 sind zwar die Ortsnamen von Lappland nicht oft zur Sprache gekommen,

11 doch hat man in aller Stille (dennoch) auch bei uns

12 diesen Bereich des Umweltschutzes gepflegt,

13 vor allem seit den sechziger Jahren immer programmatischer und intensiver.

14 Die Ergebnisse werden in der Öffentlichkeit langsam sichtbar.

15 Mit den Problemen des Ortsnamengutes in Lappland

16 hat sich in Finnland von jeher das Landesvermessungsamt

17 in seiner KARTOGRAPHISCHEN ARBEIT beschäftigen müssen.

.....

18 Die verschiedenen Ausdrucksformen des stark expansiven LapplandInteresses

19 - die wissenschaftliche Forschung, die Ausnutzung der Energiequellen,

20 die Neusiedlung, das EINÖDWANDERN, der Massentourismus -

21 haben auch in die äussersten Ecken (hintersten Winkel) von Lappland SÜDLICHTER gebracht

22 und für eine Herausforderung an die Kenner des Namengutes gesorgt:

23 wie heissen die Gegenden (Orte), wo man sich befindet?

24 Andererseits sieht man nunmehr ein,

25 dass DER LAPPISCHE (VOLKS)STAMM ein Recht hat

26 auf seine eigene Sprache, deren Schutz und ENTwickLUng.

27 Die Namenpflege gehört als wesentlicher Teil zu dieser Arbeit:

28 in den Namen sind sowohl das praktische Sprachkapital

29 als auch die gefühlsmässig erfassten TRADITIONELLEN WERTE vertreten,

30 deren Behandlung nicht mit einem Achselzucken abgetan werden kann.

reich lässt sich eine Eins-zu-Eins-Entsprechung am seltensten aufrechterhalten, s. z.B. Zeile 23: in der AS ein Fragesatz, eine Wortfrage (wörtl.: 'auf welch-namigen Orten ist man in Bewegung?'). In der ZS befriedigt 'sich befindet' nicht ganz: der Ausdruck ist zu statisch, welcher Eindruck vielleicht durch ein eingeschobenes 'jeweils' abgeschwächt werden könnte. Zeile 2 enthält die nur in der AS vorhandene Komparation des Nomens, für die sich in der ZS hier ein recht gutes Äquivalent 
anbietet. Interessanter als diese syntaktische Eigenart ist hier die Perspektive, die Blickrichtung der beiden Sprachen: die AS sieht etwas aus der Tiefe an die Oberfläche steigen, die ZS etwas aus dem Hintergrund in den Vordergrund treten. - Zeile 30 schliesslich ist ein schönes Beispiel für phraseologisch verfestigte Unterschiede im syntaktischen Bereich.

Semantik. Zunächst sei auf die Frage der Komposita aufmerksam gemacht (AS Zeile 17, 20, 29). Es ist in diesem Rahmen unmöglich, Regeln darüber aufzustellen, wann einem finnischen Kompositum im Deutschen ebenfalls ein Kompositum entspricht, wann - bei zweigliedrigen Komposita - ein Adjektiv + Substantiv. Wir wollen uns nur das Wort eräretkeily (Z. 20) näher ansehen. Die deutsche Entsprechung 'Einödwandern' (wie übrigens auch 'Neusiedlung' für uudisasutus) hat zunächst eine viel geringere Frequenz, ist fast ein Übersetzungsexotikum. Das gilt auch für das ebenfalls mögliche synonyme 'Wildmarkwandern, Wandern in der Wildmark'. Hier ergibt sich für den deutschen Leser des Textes ein Informationsverlust, dem auch der Übersetzer nicht abhelfen kann. Ähnlich verhält es sich mit dem Wort heimo 'Stamm, Volksstamm', zumal hier im Deutschen ideologische Assoziationen wach werden können, die im Finnischen fehlen. Vielleicht wäre in der ZS hier 'die Lappen' angebrachter, da auch 'das lappische Volk' nicht befriedigt. Obwohl der Textautor selbst die Benutzung des modischen saamelainen usw. vermeidet, möchte ich an dieser Stelle Sinn und Notwendigkeit der Einführung eines neuen Lexems 'der Same' statt 'der Lappe', 'samisch' statt 'lappisch' im Deutschen in Frage stellen. Und das unabhängig davon, dass bereits ein Homonym im Deutschen vorhanden ist, samisch das Adj. zu Samos ist, Samland, Samländer, samländisch auf die ostpreussische Landschaft hinweisen etc. In bestimmten Kommunikationssituationen wird sich der Hinweis auf diese Eigenbenennung der Lappen ohnehin ergeben.

AS Zeile 26: die Übersetzung von kehittäminen mit 'Entwicklung' ist nicht voll äquivalent. Genauer könnte diese Stelle lauten: 'dass die Lappen ein Recht darauf haben, eine eigene 
Sprache zu besitzen, sie zu schützen und zu entwickeln'. Dann lässt jedoch der Stil in der ZS jene sachlich knappe Prägnanz vermissen, die für einen Fachtext typisch ist.

Es gibt in dieser Textstelle einen Ausdruck, der m.E. als solcher unübersetzbar ist, AS 21: lantalaiset. Dieses alte Wort - von den Lappen pejorativ für die Finnen bzw. die Nichtlappen verwendet und etymologisch auf an. land 'Land' zurückgehend - erweckt bei Finnen heute leicht Assoziationen, die mit fi. lanta 'Mist, Dung' zusammenhängen (also lantalaiset etwa 'Mistleute'). Der Übersetzer erinnert sich an Parallelfälle in anderem Milieu, z.B. 'Bleichgesichter' (Bezeichnung der Indianer für die Weissen) oder - moderner 'Itaker' (umgangssprachliche Bezeichnung für Gastarbeiter [urspr. nur Italiener] in der Bundesrepublik Deutschland). Man könnte hier 'Finnen' oder 'Fremde' übersetzen, doch hätte man dann auf jegliche Stilfärbung bewusst verzichtet. Meine Wahl 'Südlichter' begründe ich wie folgt: der Ausdruck entspricht inhaltlich dem finnischen erstaunlich gut, denn die lantalaiset, das sind die südlich von Lappland ansässigen, etwas abfällig so titulierten Leute. Negativ d.h. schlecht an dieser deutschen Entsprechung ist, dass sie ganz modern und auch nur den Deutschen vertraut ist, die die Presse und politische Diskussion in der Bundesrepublik verfolgen. Da kam nämlich zunächst wohl im Wahlkampf - die Bezeichnung 'Nordlichter' für die Leute nördlich der Rhein-Main-Linie auf, die dann ihrerseits scherzhaft mit der Formulierung 'Südlichter' konterten.

Schliesslich könnte man hier aber auch eine Neubildung wagen: Finnski, $-s, m$. in Anlehnung an eine andere im Deutschen geläufige, leicht abwertende Nationalitätsbezeichnung, Russki, -s.

Stil. Stilistisch ist dem Autor wie dem Übersetzer bei Texten der vorliegenden Art relativ wenig Spielraum gelassen. Das stellt an den Übersetzer die Anforderung, dass er das diesbezügliche Ausdruckspotential der AS als solches erkennt und jenes in der ZS beherrscht. Z.B. Zeile 1: koskeva besser 'für' als 'betreffend' in einer Klischeeformulierung. Zeile 16 meillä 'in Finnland': eine typische Häufigkeitsrestriktion. Und schliess- 
lich Zeile 18: hier könnte es an sich in der ZS auch wörtlicher heissen 'Das stark angewachsene Lappland-Interesse mit seinen verschiedenen Ausdrucksformen'. Bei der Übersetzung des im Finnischen in dieser Stellung so frequenten Partiz. Perf. sollte man sich jedoch stets überlegen, ob die temporale Bedeutungsnuance wirklich wesentlich die der Vergangenheit ist, zumal das deutsche Partiz. Prs. tempusmässig nicht ausschliesslich auf die Bezeichnung der Gegenwart festgelegt ist. Dass hier dann doch ein Adjektiv anstelle eines Partizips gewählt wurde, hängt mit den sog. Natürlichkeitsbedingungen der ZS zusammen, wobei der sprachliche Rhythmus eine nicht unwesentliche Rolle zu spielen scheint.

Die Aussagefähigkeit einer Rückübersetzung des deutschen Textes ins Finnische muss angezweifelt werden; W. Wilss (Übersetzungswissenschaft, S. 190) meint sicher zu Recht, aus einer besseren Rückübersetzbarkeit einer Übersetzung könne nicht generell auf einen höheren Adäquatheitsgrad sondern eher auf eine bestimmte Übersetzungsprozedur bzw. deren Folge geschlossen werden. Im vorliegenden Fall besteht die Wahrscheinlichkeit, dass eine Rückübersetzung oberflächenstrukturell etwas unterschiedlich ausfallen würde.

11. Jede uralische Sprache lässt sich auf ihre Weise leicht oder schwer in eine nichturalische Sprache übersetzen. Meine eigene Erfahrung würde darauf hindeuten, dass es für den Translator deutscher Muttersprache schwerer ist, aus dem Finnischen zu übersetzen als aus dem Ungarischen. Dies dürfte in erster Linie syntaktische Gründe haben. Das gesamte Feld der Verbalkonstruktionen im Finnischen, die relative Häufigkeit der Satzäquivalente, die im Finnischen im Gegensatz zum Ungarischen weitgehend erhaltene uralische Sicht der Rektionen - dies sind einige Schwerpunkte. Das Ungarische verfügt heute über ein ausgeprägtes System von Präfixverben, die im alten Ungarisch noch geläufigen Satzäquivalente sind Nebensätzen gewichen und die Sprache war überhaupt nichturalischen Einflüssen gegenüber zugänglicher als das Finnische. Dieses Problem ist äusserst komplex und kann hier nur angeschnitten werden. An sich geht es dabei um Ähnliches, was durch Wolfgang Schlachter und Jenő Kiss unter dem Thema 'Schwie- 
rigkeiten beim Erlernen des Ungarischen und Finnischen" (Acta Linguistica 1976) verdienstvoll untersucht worden ist. Die Autoren kamen zu dem Ergebnis, dass die finnische Sprache auf der orthographisch-phonetischen Ebene für den deutschen Muttersprachler leichter erlernbar ist als die ungarische Sprache, dass die Schwierigkeiten auf der morphologischen Ebene in beiden Sprachen gleich gross sind, während das Finnische seiner Syntax wegen bedeutend schwerer ist als das Ungarische.

12. Die Verwendung interlinearer deutscher Übersetzungen bei der Veröffentlichung finnisch-ugrischer Sprachproben ist eine gute alte Sitte, sicher auch weiterhin brauchbar. Eine solche Übersetzung veraltet nicht oder kaum und ist auch leicht für einen Leser verständlich, dessen Erstsprache nicht das Deutsche ist. Die Zugeständnisse, die bei solchen Übertragungen (z.B. aus dem Ostjakischen) an die Normen des Deutschen gemacht werden, dürfen jedoch nicht auf Kosten der Verständlichkeit gehen. Diese Gefahr besteht einmal in der Reihenfolge der Wortstellung, besonders aber bei der Nachahmung der Wortbildung und Komposita-Gebilde des Ostjakischen. Bleibt der Inhalt voll verständlich, ist gegen ein solches Verfahren innerhalb der Finnougristik nichts einzuwenden. Im Hinblick darauf kann man mit Ortega y Gasset sagen:

"Ich stelle mir eine Art von Übersetzung vor, die unschön ist, wie es die Wissenschaft immer ist, die keine literarische Anmut für sich in Anspruch nimmt, die nicht leicht zu lesen ist, die aber ganz klar ist, auch wenn diese Klarheit eine Menge von Fussnoten erfordert. Es ist nötig, dass der Leser im voraus wisse, dass, wenn er eine Übersetzung liest, er kein vom literarischen Standpunkt schönes Buch liest, sondern dass er ein ziemlich beschwerliches Hilfsmittel benützt."

Da die Übersetzungswissenschaft einmal den Translationsprozess als solchen, zum andern die sprachenpaarbezogene translatorische Performanz und auf diesem Wege auch die translatorische Kompetenz im allgemeinen untersucht, als interlinguale Sprachverstehenskompetenz und als sprachliche Reproduktionskompetenz, ist sie durchaus fähig, die Ausgangs- 
und Ansatzpunkte zu erhellen, von denen jegliche kontrastive Forschung auszugehen hat.

INGRID SCHELLBACH

\section{BIBLIOGRAPHIE (in Auswahl)}

J. C. Catford, A Linguistic Theory of Translation. An Essay in Applied Linguistics. London 1965.

E. Coseriu, Das Phänomen der Sprache und das Daseinsverständnis des heutigen Menschen. In: E. Coseriu, Sprache, Strukturen und Funktionen. Tübingen 1970, 111-136.

F. Güttinger, Zielsprache. Theorie und Praxis des Übersetzens. Darmstadt 1969.

M. A. K. Halliday, Comparison and Translation. In: M. A. K. Halliday, A. McIntosh, P. Strevens (Eds.), The Linguistic Sciences and Language Teaching. London 1964, 111-134.

Hornung-Kretschmar-Ortmann-Wüsteneck, Die Übersetzung wissenschaftlicher Literatur aus dem Russischen ins Deutsche. Leipzig 1974.

G. Jäger, Translation und Translationslinguistik. Halle/S. 1975.

R. Jakobson, On Linguistic Aspects of Translation. In: R. A. Brower (Ed.), On Translation. New York 1966.

J. Juhász, Probleme der Interferenz. München 1970.

R. W. Jumpelt, Die Übersetzung naturwissenschaftlicher und technischer Literatur. Berlin-Schöneberg 1961.

O. Kade, Zufall und Gesetzmässigkeit in der Übersetzung. Leipzig 1968. (Beihefte zur Zeitschrift Fremdsprachen. 1.)

O. Kade, Kommunikationswissenschaftliche Probleme der Translation. Grundfragen der Übersetzungswissenschaft. In: Beihefte zur Zeitschrift Fremdsprachen. 2. Leipzig 1968, 3-20.

O. Kade (Hrsg.), Vermittelte Kommunikation, Sprachmittlung, Translation. Übersetzungswissenschaftliche Beiträge 1. Leipzig 1977.

Kapp, V. (Hrsg.), Übersetzer und Dolmetscher. UTB 325. Heidelberg 1974.

W. Koller, Eine linguistische Theorie des Übersetzens. In: Moderna Språk 1969, 264-70. Stockholm 1969.

W. Koller, Grundprobleme der Übersetzungstheorie. München 1972.

W. Koller, Übersetzungswissenschaft. In: FoL 5. 1972, 194-221.

A. Malblanc, Stylistique comparée du français et de l'allemand. Essai de représentation linguistique comparée et étude de traduction. Paris 1966.

H. Martin, Untersuchungen zur sprachlichen Interferenz auf der Grundlage finnlanddeutschen Materials. Turku 1973.

G. Mounin, Die Übersetzung. Geschichte, Theorie, Anwendung. München 1967.

E. A. Nida, Science of Translation. In: $\operatorname{Lg~45,~1969,~483-498.~}$ 
J. Ortega y Gasset, Elend und Glanz der Übersetzung. (Publ. in: H. J. Störig, s.u.)

F. Paepcke, Verstehen und Übersetzen. In: LAnt 2. 1968, 329-351.

F. Paepcke, Gemeinsprache, Fachsprache und Übersetzen. CEBAL 3. Kopenhagen 1975.

K. Reiss, Möglichkeiten und Grenzen der Übersetzungskritik. Kategorien und Kriterien für eine sachgerechte Beurteilung von Übersetzungen. München 1971.

K. Reiss, Texttyp und Übersetzungsmethode. Kronberg/Ts. 1976.

H. J. Störig (Hrsg.), Das Problem des Übersetzens. Stuttgart 1969.

L. Tarnóczi, Fordítókalauz. A szakirodalmi fordítás elmélete és gyakorlata. Budapest 1966.

L. Tarnóczi, Fordítástechnika (Müszakigazdasági fordítások idegen nyelvböl magyarra). Budapest 1972.

J. P. Vinay et J. Darbelnet, Stylistique comparée du français et de l'anglais. Méthode de traduction. Paris 1966.

M. Wandruszka, Sprachen vergleichbar und unvergleichlich. München 1969.

U. Weinreich, Languages in Contact. Den Haag/Paris $1974^{8}$.

W. Wilss, Übersetzungswissenschaft. Probleme und Methoden. Stuttgart 1977. 